OPEN ACCESS

Edited by:

Jean-Philip Piquemal,

Sorbonne Universités, France

Reviewed by:

Gerardo Andres Cisneros,

University of North Texas,

United States

Patrick Senet,

Université de Bourgogne, France

*Correspondence:

Giovanni La Penna

giovanni.lapenna@cnr.it

Specialty section: This article was submitted to Theoretical and Computational

Chemistry,

a section of the journal

Frontiers in Chemistry

Received: 22 September 2017 Accepted: 08 January 2018 Published: 30 January 2018

Citation:

La Penna G and Chelli R (2018)

Structural Insights into the Osteopontin-Aptamer Complex by Molecular Dynamics Simulations.

Front. Chem. 6:2.

doi: 10.3389/fchem.2018.00002

\section{Structural Insights into the Osteopontin-Aptamer Complex by Molecular Dynamics Simulations}

\author{
Giovanni La Penna ${ }^{1 *}$ and Riccardo Chelli ${ }^{2}$ \\ ${ }^{1}$ Istituto di Chimica dei Composti Organometallici, Consiglio Nazionale delle Ricerche (CNR), Florence, Italy, ${ }^{2}$ Dipartimento di \\ Chimica, Università di Firenze, Florence, Italy
}

Osteopontin is an intrinsically disordered protein involved in tissue remodeling. As a biomarker for pathological hypertrophy and fibrosis, the protein is targeted by an RNA aptamer. In this work, we model the interactions between osteopontin and its aptamer, including mono- $\left(\mathrm{Na}^{+}\right)$and divalent $\left(\mathrm{Mg}^{2+}\right)$ cations. The molecular dynamics simulations suggest that the presence of divalent cations forces the $\mathrm{N}$-terminus of osteopontin to bind the shell of divalent cations adsorbed over the surface of its RNA aptamer, the latter exposing a high negative charge density. The osteopontin plasticity as a function of the local concentration of $\mathrm{Mg}$ is discussed in the frame of the proposed strategies for osteopontin targeting as biomarker and in theranostic.

Keywords: osteopontin, aptamers, intrinsically disordered proteins, molecular models, ion condensation

\section{INTRODUCTION}

Osteopontin (OPN) belongs to the class of matricellular proteins involved in tissue remodeling (Frangogiannis, 2012; Kahles et al., 2014). After injuries in tissues, cell replication is induced by many extracellular factors that become over-expressed. OPN expression increases when cells replication is induced and when collagen extracellular matrix has to be reconstructed to stabilize the cells in the new tissue. Knock-out of OPN gene (SPP1 in human) eliminates the reconstruction of collagen. An increase of OPN concentration in serum is a clear indication of myocardial remodeling, both after myocardial infarction and in case of pathological cardiac hypertrophy and fibrosis.

OPN is the target of several therapies that have been proposed to interfere with the signaling process activating or modulating cell-cell or cell-matrix interactions. A proposed theranostic agent is based on the specific interactions between osteopontin and an RNA aptamer (Mi et al., 2009) (OPN-R3, hereafter). These RNA aptamers can be easily synthesized in vitro and supported over gold nanoparticles (Liu and $\mathrm{Lu}, 2006$ ) containing fluorescent probes or dyes. The nanoparticles can therefore be used to monitor the concentration of OPN in serum or to deliver drugs in the extracellular region where the OPN concentration is pathologically high.

OPN belongs to the class of intrinsically disordered proteins (IDPs). The protein of 300 aminoacids has no three-dimensional structure in water (Kurzbach et al., 2013; Platzer et al., 2015). The many interactions between OPN and other proteins and cofactors indicate that the lack of structure enables OPN to perform different functions (Sodek et al., 2000; Mazzali et al., 2002). Among these interactions we mention: (i) integrin receptors with the RGD domain (Christensen et al., 2010); (ii) CD44 domain and collagen, with highly negative polysaccharides involved (Ponta et al., 2003); (iii) post-translational modifications activating different biochemical pathways (Weber et al., 2002), including biomineralization (Li and Wang, 2012); (iv) interactions 
with cations ( $\mathrm{Ca}, \mathrm{Mg}$ ) modulating $\mathrm{OPN}$ activity ( $\mathrm{Hu}$ et al., 1995); (v) OPN antibodies inhibiting the rheumatoid arthritis propagation (Du et al., 2008) (PDB 3CXD). We shall indicate this framework of diverse interactions as OPN plasticity (Peysselon et al., 2011).

The role of IDPs in biomineralization has been reported (Kalmar et al., 2012) and OPN shares this property with many IDPs (Hunter et al., 2010). The interaction with $\mathrm{Ca}^{2+}$ cations has been hypothesized in the N-terminus of OPN, characterized by a significant density of $\mathrm{D} / \mathrm{E}$ residues (single-letter aminoacid coding is mostly used, hereafter). The protein contains, in the post-processed 17-300 chain, $47 \mathrm{D}, 24 \mathrm{E}, 18 \mathrm{~K}$, and $9 \mathrm{R}$, for a total charge at physiological $\mathrm{pH}$ of -44 . The high polarization of charge in OPN (the charge of first 100 residues is -26) indicates that the interactions of OPN with macromolecular partners is associated to a collective rearrangement of the $\mathrm{N}$-terminus consequent to the approach of the target molecule by OPN.

As for the binding of OPN to RNA, two possible arrangements of the approaching OPN random coil can be hypothesized, as drawn in Figure 1.

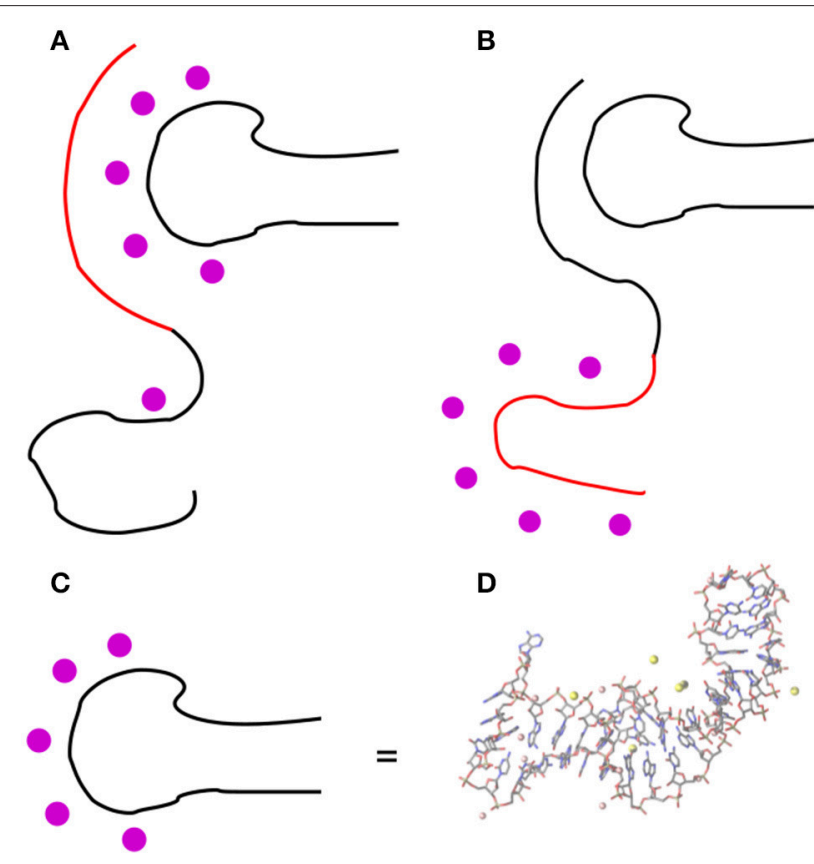

FIGURE 1 | Two possible arrangements of the OPN coil in the OPN-(OPN-R3) complex: (A) the negatively charged $\mathrm{N}$-terminus (in red) forms an extended array of electrostatic interactions (like salt bridges) with the RNA phosphate groups, mediated by mono- and divalent cations (magenta circles); (B) the negatively charged $\mathrm{N}$-terminus is repelled by RNA and the $\mathrm{C}$-terminus is forced for non-electrostatic interactions with RNA. In (D) the RNA drawing of OPN-R3 (C) is expanded into the atomic structure obtained as the final configuration of OPN-R3 simulated for 23.4 ns with $\mathrm{MgCl}_{2}$ (see also Figure S4). RNA is represented as sticks; $\mathrm{C}$ is gray, $\mathrm{N}$ blue, $\mathrm{O}$ red; $\mathrm{H}$ atoms are not displayed; $\mathrm{Mg}$ and $\mathrm{Na}$ ions within $0.5 \mathrm{~nm}$ from $\mathrm{OP}$ are represented as pink and yellow spheres, respectively. Bond and atomic radii are arbitrary. The stem-loop RNA region (11-24) is on the left-hand side and the RNA termini (1-2 and 34-40) are on the top-right. The VMD program (Humphrey et al., 1996) is used for all the molecular drawings.
In the first hypothesis, the negatively charged $\mathrm{N}$-terminus of OPN forms an extended array of electrostatic interactions with mono- and divalent cations more or less adsorbed on the RNA molecule (the phosphate groups). In the second hypothesis, the negatively charged N-terminus is repelled by RNA and the interactions between OPN and RNA are non-electrostatic, even though they can still involve the cations adsorbed on the RNA molecule.

The interactions between OPN and heparin, the latter a polysaccharide with high negative charge density, have been recently studied by NMR and EPR techniques (Kurzbach et al., 2014, 2017). These data are consistent with the formation of a patch of complementary charges, with an unfolding-uponbinding mechanism that releases the compaction of OPN in the absence of any partner. This kind of assembly process resembles that drawn in Figure 1B. On the other hand, all NMR and EPR studies performed so far are in the absence of divalent cations.

In this work, we aim at providing models to understand the OPN plasticity, with the specific goal of understanding how OPN interacts with its aptamer OPN-R3 (Mi et al., 2009), in the presence of naturally occurring divalent cations. Therefore, still in the frame of the electrostatic hypothesis that is generally assumed for IDPs, we exploit the other possible mechanism (Figure 1A), on the basis of the expected strong effect of divalent cations in the RNA function.

Despite the limited time-length (55 ns) of molecular dynamics simulations performed for the large macromolecular complex (more than 500,000 atoms, including the model of ionic atmosphere), the structural models reported here provide a possible alternative frame compared to that observed with no divalent cations, and assist to a better prediction of the interactions of OPN when it is bound to the aptamer and to the theranostic nanoparticle that supports the aptamer.

\section{METHODS}

We built 6 initial configurations for the OPN-(OPN-R3) complex with a low number of intermolecular contacts (see Figure S1). Explicit water molecules and counterions were included. All the 6 initial structures were simulated with molecular dynamics (MD) in the NPT statistical ensemble at $T=300 \mathrm{~K}$ and $P=0$ bar, in a range of physiological salt concentrations conditions, for 10 ns. Two of these configurations were simulated for 55 ns. The system size is summarized in Table 1. The size, in terms of number of atoms, is significant because of the requirement of modeling the ionic atmosphere including divalent cations and because of the size of the initial models for the disordered OPN protein. This size prevents the collection of adequately long MD simulations (likely $1 \mu \mathrm{s}$ ) and of more independent or statistically combined trajectories (likely more than one hundred). Therefore, we concentrate the modeling to discriminate between the two different complex topologies described in Figure 1.

All the details of the construction and simulations are reported in the Supplementary Material. Here, we summarize only the most important points. The AMBER PARM99 force field (Want et al., 2000) was used in all simulations performed with the 
LAMMPS code (Plimpton, 1995). As for cations, we used a recent force field for divalent cations (Duarte et al., 2014), in addition to the usual parametrization of $\mathrm{NaCl}$ (Aaqvist, 1990). This force field reduces the charge density over the divalent cation, thus avoiding the usual problem of negatively charged

TABLE 1 | Number of atoms in the simulated systems.

\begin{tabular}{|c|c|c|c|c|}
\hline Trajectory & Species & Part 1 & Part 2 & Part 3 \\
\hline \multirow[t]{5}{*}{ OPN-R3 } & $\mathrm{Na}$ & 243 & - & 243 \\
\hline & $\mathrm{Mg}$ & 20 & - & 0 \\
\hline & $\mathrm{Cl}$ & 244 & - & 204 \\
\hline & wat & 108,645 & - & 108,705 \\
\hline & total & 327,842 & - & 327,842 \\
\hline \multirow[t]{4}{*}{1} & $\mathrm{Na}$ & 366 & 384 & 406 \\
\hline & $\mathrm{Mg}$ & 20 & 11 & 0 \\
\hline & wat & 172,307 & 172,298 & 172,287 \\
\hline & total & 523,319 & 523,247 & 523,159 \\
\hline \multirow[t]{4}{*}{2} & $\mathrm{Na}$ & 366 & 368 & 406 \\
\hline & $\mathrm{Mg}$ & 20 & 19 & 0 \\
\hline & wat & 172,279 & 172,278 & 172,287 \\
\hline & total & 523,235 & 523,227 & 523,159 \\
\hline \multirow[t]{4}{*}{3} & $\mathrm{Na}$ & 366 & 374 & 406 \\
\hline & $\mathrm{Mg}$ & 20 & 16 & 0 \\
\hline & wat & 172,281 & 172,277 & 172,261 \\
\hline & total & 523,241 & 523,209 & 523,081 \\
\hline \multirow[t]{4}{*}{4} & $\mathrm{Na}$ & 366 & 376 & 406 \\
\hline & $\mathrm{Mg}$ & 20 & 15 & 0 \\
\hline & wat & 172,314 & 172,309 & 172,294 \\
\hline & total & 523,340 & 523,300 & 523,180 \\
\hline \multirow[t]{4}{*}{5} & $\mathrm{Na}$ & 366 & 386 & 406 \\
\hline & $\mathrm{Mg}$ & 20 & 10 & 0 \\
\hline & wat & 172,250 & 172,240 & 172,230 \\
\hline & total & 523,148 & 523,068 & 522,988 \\
\hline \multirow[t]{4}{*}{6} & $\mathrm{Na}$ & 366 & 368 & 406 \\
\hline & $\mathrm{Mg}$ & 20 & 19 & 0 \\
\hline & wat & 172,294 & 172,293 & 165,893 \\
\hline & total & 523,280 & 523,272 & 503,977 \\
\hline
\end{tabular}

Atoms in OPN protein are 4,289 (284 aminoacids) and in OPN-R3 aptamer are 1,280 (39 bases). The number of chloride ions is always 323 for OPN-(OPN-R3) complex. The amount of water solvent (wat) is in molecules. (carboxylate and phosphate) groups collapsing over the highlycharged points. For long-range electrostatic interactions, we used the damped and shifted force method (Fennell and Gezelter, 2006) implemented in LAMMPS. For a critical review about this method compared to more accurate, but computationally slower, methods (see Cisneros et al., 2014).

After the construction of the OPN-R3 structure, the structure of the ionic layers around it has been simulated by MD in explicit water for about 23 ns. The analysis confirmed most of the observations reported in the literature about the structure of mono- and divalent cations around RNA stem-loop configurations. This analysis is reported in the Supplementary Material.

As for the construction of initial configurations for the OPN(OPN-R3) complex, the configuration of RNA with Mg simulated after $18 \mathrm{~ns}$ was used. This configuration, as shown by the analysis described in the Supplementary Material, has $9 \mathrm{Mg}$ cations adsorbed. These cations were kept for the construction of the initial complex samples.

A random configuration for the OPN protein was built and put in contact with the OPN-R3 selected structure. A set of non overlapping OPN-(OPN-R3) configurations was generated by using random temperature Monte Carlo trajectories (La Penna, 2003; La Penna et al., 2004) (MC-RW), summarized in the Supplementary Material. The OPN C-terminus (N284) and the RNA OPN-R3 aptamer were mutually oriented and kept fixed in space in 6 samples, differing for the initial position of the C-terminus in front of the center of the RNA stem. The entire protein chain of OPN randomly moved around the RNA molecule. For each sample, we choose the first configuration displaying a manifest contact between OPN and OPN-R3 as the starting configuration for the following MD simulation (Figure S1, Supplementary Material). Therefore, once a configuration mimicking a possible initial contact between OPN and OPN-R3 was selected within the $6 \mathrm{MC}-\mathrm{RW}$ performed, MD simulations of the OPN-(OPN-R3) complex were performed in explicit water and with explicit counterions.

The chosen $\mathrm{Mg}$ concentrations (see Table 2) are consistent with the range of concentrations for divalent cations $\left(\mathrm{Mg}^{2+}\right.$ and $\mathrm{Ca}^{2+}$ ) in the simulated body fluid (Oyane et al., 2003) used for in vitro OPN-RNA binding (Mi et al., 2009) and biomineralization studies (Kalmar et al., 2012). After performing $10 \mathrm{~ns}$ of simulation in the NPT statistical ensemble for the 6 samples, each at the 3 different salt concentrations, we performed

TABLE 2 | Equilibrium concentrations (mM) of $\mathrm{Na}$ and $\mathrm{Mg}$, and simulation times in the different samples.

\begin{tabular}{|c|c|c|c|c|c|c|c|c|c|}
\hline \multirow[b]{2}{*}{ OPN-R3 } & \multicolumn{3}{|c|}{ Part 1} & \multicolumn{3}{|c|}{ Part 2} & \multicolumn{3}{|c|}{ Part 3} \\
\hline & 121 & 10 & (10) 13.4 & - & - & - & 121 & 0 & (5) 16.2 \\
\hline 1 & 114 & 6 & (5) 6.1 & 120 & 3 & (5) 5.2 & 127 & 0 & (5) 5.0 \\
\hline 2 & 114 & 6 & (5) 5.0 & 115 & 6 & (5) 5.8 & 127 & 0 & (5) 6.0 \\
\hline 3 & 114 & 6 & (5) 8.2 & 117 & 5 & (5) 7.5 & 127 & 0 & (5) 5.2 \\
\hline 4 & 114 & 6 & (5) 5.7 & 118 & 5 & (5) 5.5 & 127 & 0 & (5) 5.0 \\
\hline 5 & 114 & 6 & (5) 5.0 & 121 & 3 & (5) 5.4 & 127 & 0 & (5) 5.2 \\
\hline 6 & 114 & 6 & (5) 5.5 & 115 & 6 & (5) 5.7 & 127 & 0 & (5) 6.0 \\
\hline
\end{tabular}

Equilibration times (within brackets) and sampling times are in ns. Volume fluctuations in the last 5 ns of all simulations are within $0.1 \%$. 
longer simulations (55 ns) for two selected samples and ionic environments.

\section{RESULTS AND DISCUSSION}

\subsection{OPN-R3 Aptamer}

The settling of the ionic atmosphere around the stem-loop structure of OPN-R3 (see Supplementary Material), showed that a high degree of screening of RNA is performed by both $\mathrm{Na}$ condensation (within $3 \mathrm{~nm}$ ) and $\mathrm{Mg}$ adsorption over the RNA surface (within $0.28 \mathrm{~nm}$ ). The analysis shows that $\mathrm{Mg}$ replaces $\mathrm{Na}$ in the condensation layer, but the $\mathrm{Mg}$ ions are more structured around the phosphate groups of RNA.

The analysis of the OPN-R3 aptamer simulations, shows that, as expected, the simulation time is not long enough to sample all the structural changes occurring in the RNA fragment during the stabilization of the layer of cations (see discussion of the rootmean square deviation and solvent accessible surface area). We remark that the aim of the model is to probe the general effect of the formed layer on the protein-RNA interactions, regardless of specific interactions that can be adequately sampled with atomistic models of this size only using hundreds of replica in the $\mu$ s time-scale.

Summarizing, the RNA molecule adsorbs $8 \pm 2$ divalent cations and $2 \pm 1 \mathrm{Na}$ cations, on average. The comparison of radial distribution function for OP-M pairs (OP being the nonphosphodiester $\mathrm{O}$ atom of RNA phosphate groups) with that for all pairs $\mathrm{X}-\mathrm{M}$ shows that this number is dominated by OP contacts when $\mathrm{M}$ is $\mathrm{Mg}$, but there is a significant difference when $\mathrm{M}=\mathrm{Na}$. Even when $\mathrm{Mg}$ is absent, the number of $\mathrm{X}$ adsorbed $\mathrm{Na}$ is $6 \pm 2$, while that for OP-adsorbed $\mathrm{Na}$ is $3 \pm 2$. This difference means that $\mathrm{Na}$ ions are not really adsorbed, while there is exchange between the first shell and farther shells, allowing short-range interactions with groups different from the phosphate groups. This observation is consistent with a loose structure of short-range $\mathrm{Na}$ shells. On the other hand, in case of $\mathrm{Mg}$, the number of adsorbed ions measures the extent of ion adsorption by the phosphate groups as an additional information to the ion condensation, the latter occurring within the larger distance of $3 \mathrm{~nm}$ (see Supplementary Material).

As a consequence of the ionic behavior summarized above (see Supplementary Material for a more detailed discussion), most of the screening contribution due to $\mathrm{Mg}$ is made by phosphateadsorbed $\mathrm{Mg}$ ( 8 cations over 9 , see Supplementary Material). This adsorption is a very slow process, as already noticed in 100-ns long simulations (Casalino et al., 2017). Na is more diffuse within the distance of about $3 \mathrm{~nm}$, and the phosphate-adsorbed $\mathrm{Na}$ ions provide a small contribution to the screening positive charge (2 over $22 \mathrm{Na}$ ions within $3 \mathrm{~nm}$ ). The structure of these layers will serve as a reference for the comparison with the OPN-aptamer complex described in the following.

\section{2. (OPN-R3)-OPN Complex}

As for (OPN-R3)-OPN complex, the time-evolution of different types of contacts and radial distribution functions have been analyzed. The time-length of 10 ns sampled for 6 models, allows the settling of the ionic atmosphere around each complex.
On a longer time-scale (55 ns), sampled for 2 of the models, an initial stage of wrapping of the protein around the RNA fragment is clearly observed (see below). The simulation of a complete wrapping process, as well as of the possible equilibrium between wrapped and unwrapped configurations, is beyond the possibilities of these atomistic models. Therefore, we focus only on discriminating among the two mechanisms described, respectively, in Figures 1A,B.

\subsubsection{OPN-Aptamer Contacts}

We first measured the number of OPN-RNA non specific contacts using a set-up already used to evaluate crystal and solution structures of several protein-RNA complexes. Then, the number of ions $\mathrm{M}$ (with $\mathrm{M}=\mathrm{Na}, \mathrm{Mg}$ and $\mathrm{Cl}$ ) is used to eventually correlate time evolution of non specific contacts with the adsorption/release of counterions from the short-range layer discussed for RNA in the absence of the OPN protein partner. Similarly to the analysis of RNA, we also analyze the location of adsorbed cations, selecting $\mathrm{O}$ atoms in negatively charged groups of OPN protein ( $\mathrm{O} \delta$ of $\mathrm{D}$ and $\mathrm{O} \epsilon$ of $\mathrm{E}$ ). These $\mathrm{O}$ atoms will be indicated as OD and OE, respectively, hereafter.

As for specific O-M contacts, the $d_{0}$ value (see Equation 1 in Supplementary Material) is the same used for RNA $(0.28 \mathrm{~nm})$, because the position of the first peak in the radial distribution function of OP-M distance does not change when OPN is added. The same effect holds for $\mathrm{O}$ of carboxylate groups in OPN. As for the first evaluation (non specific contacts), the work reported in (Lejeune et al., 2005) was used as a reference. In that work, the $d_{0}$ distance was $0.5 \mathrm{~nm}$, involving any protein and RNA atom pairs, and a deep analysis was performed for 49 structure entries in the protein data-bank (PDB). We measured $C N$ with $d_{0}=$ $0.5 \mathrm{~nm}$ and for pairs involving non-hydrogen atoms of protein and RNA, respectively, for the 41 complexes, among the 49 PDB entries, for which it is possible to reconstruct the entire unit cell. This construction was performed with the VESTA program (Momma and Izumi, 2011). Among the 41 structures a further selection must be made to compare the number of contacts with the OPN-aptamer 1:1 complex investigated in this work. We selected structures where the ratio $R=n_{a a} / n_{n t}$ between the number of aminoacids in protein $\left(n_{a a}\right)$ and the number of nucleotides in the RNA partner $\left(n_{n t}\right)$ is in the range of the ratio that holds for the OPN-aptamer 1:1 complex, $R=284 / 40 \simeq 7$. Therefore, we selected the 26 structures with ratio between 3 and 15 . The $C N$ value obtained for these 26 structures is then normalized to $C N_{n}=C N /\left(n_{a a}+n_{n t}\right)$, i.e., is divided by the total number of residues $n_{a a}+n_{n t}$ in the unit cell. The periodic boundary conditions of the unit cell were used to compute the distances in the evaluation of $\mathrm{CN}$ in Equation 1 (Supplementary Material). The minimal value for $C_{n}$ in this set of structures is 0.002 and the maximal is 0.07 . Among this data-set, the unique structure of a protein-aptamer complex is the structure 1OOA, with $C N_{n}=0.02$. Hereafter, we shall use this value as the $C N_{n}$ minimal value for the protein-RNA binding.

In left panels of Figure 2, the time evolution of $\mathrm{CN}_{n}$ in the six simulations of OPN-aptamer complex is displayed, while the time evolution of specific O-M contacts $\left(n_{1}\right.$, see Supplementary Material) is displayed in Figure 3. 

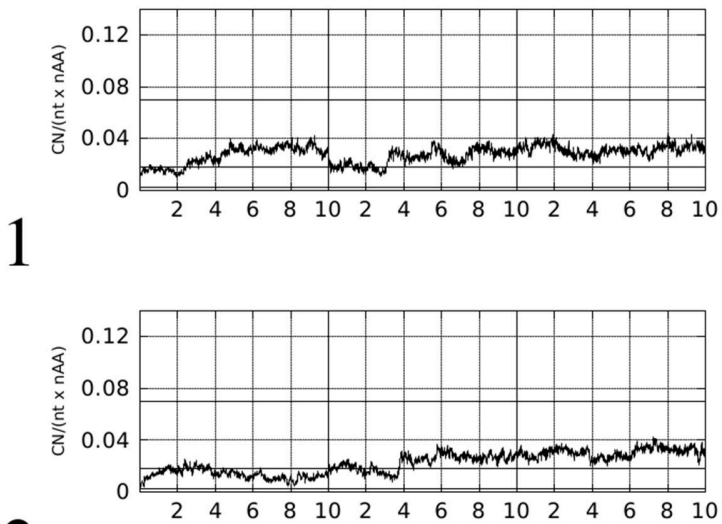

2
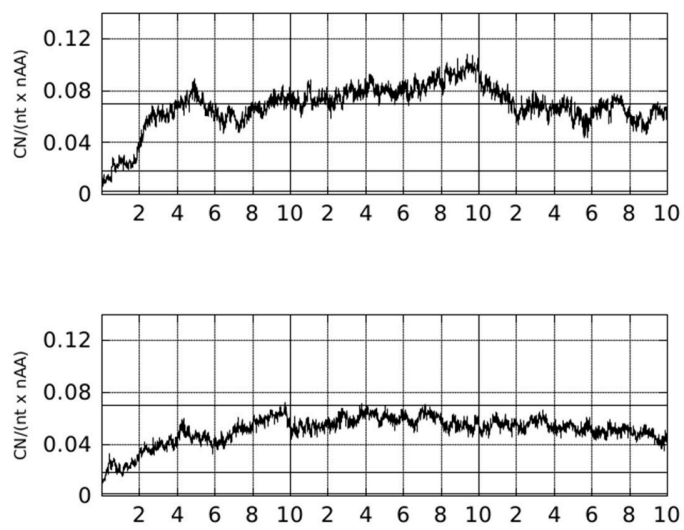

4
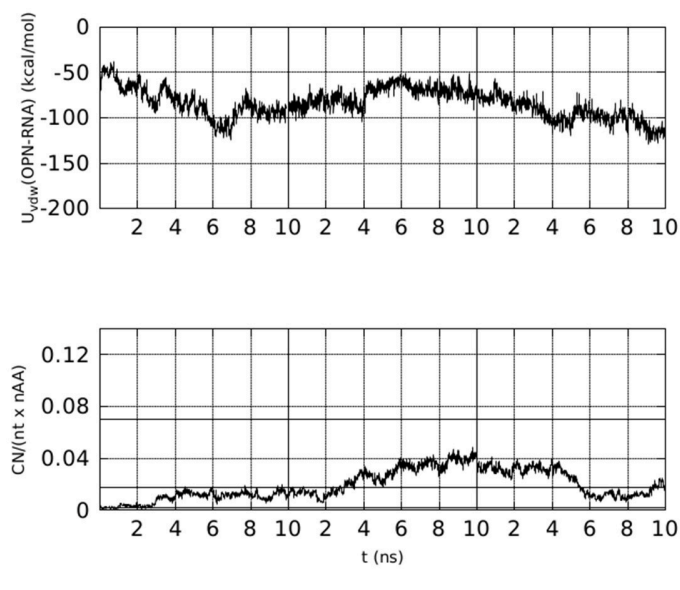
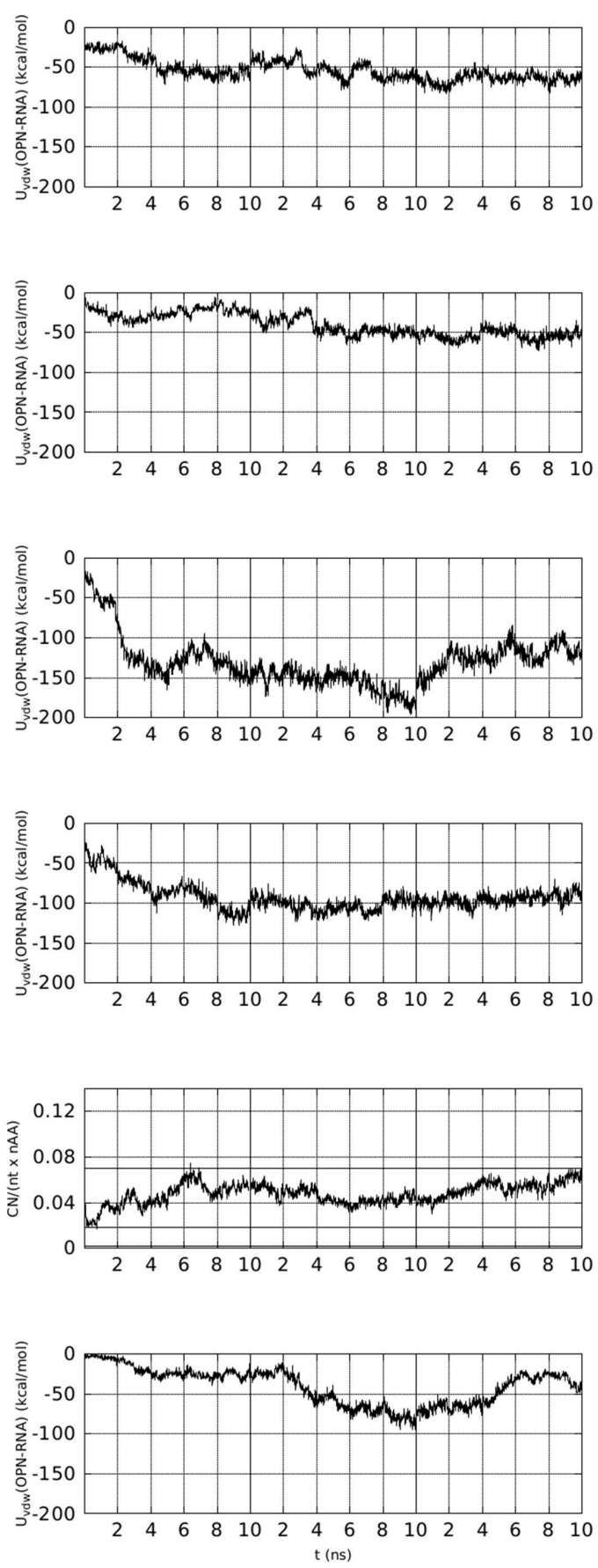

FIGURE 2 | Left: time evolution, within the first 10 ns of simulation, of the number of contacts (CN $N_{n}$, see text for details) involving OPN and OPN-R3 non-hydrogen atoms $\left(d_{0}=0.5 \mathrm{~nm}\right)$. Right: time evolution of Lennard-Jones contributions to OPN-(OPN-R3) energy $\left(U_{v d w}\right)$. The vertical lines separate simulations with decreasing (from left to right) concentration of Mg. The horizontal lines in left panels indicate the range of values obtained with the identified sub-set of protein-RNA complexes in $\mathrm{PDB}$, with the line at 0.02 being related to PDB record $100 \mathrm{~A}$ (protein-aptamer complex).

Left panels of Figure 2 display two features concerning the wrapping of OPN around the RNA molecule. The first feature is that in all cases there is a rapid increase in the number of contacts during the first half (5 ns) of the trajectory. This increase, with the exception of trajectory $\mathbf{5}$, starts from values lower than the threshold displayed by the aptamer complex reported in the PDB (1OOA, 0.02), and ends within the first part of simulations at values higher than the threshold, with the 


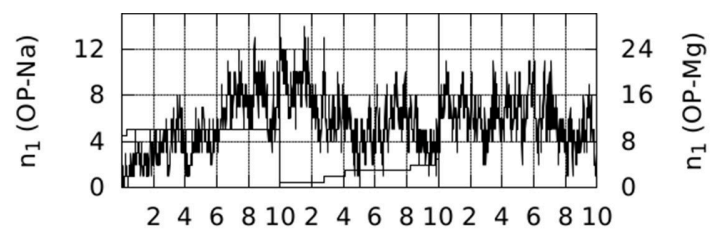

1

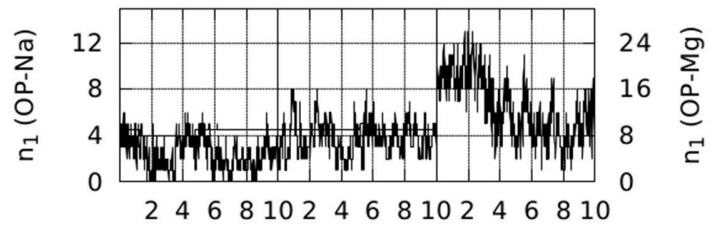

2

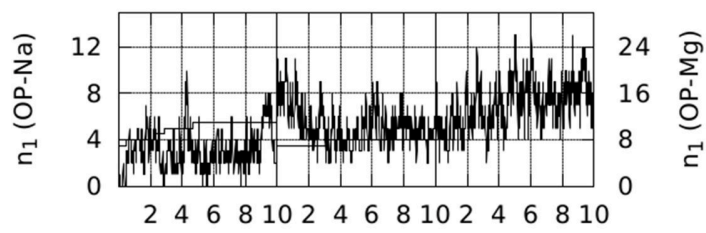

3

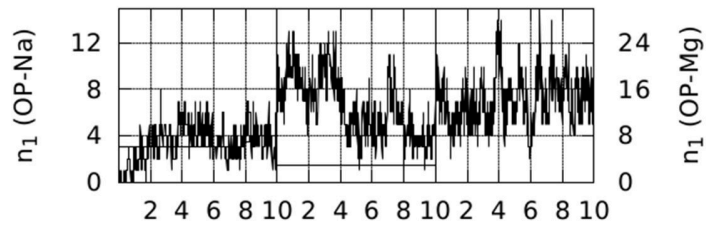

4

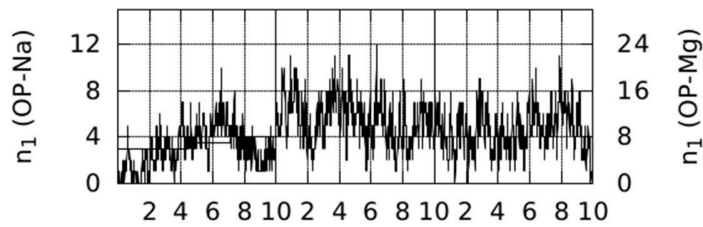

5

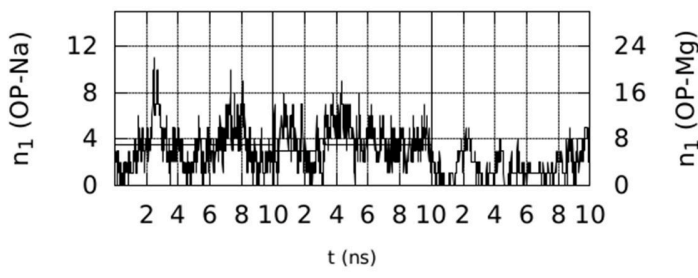

6

FIGURE 3 | Time evolution, within the first 10 ns of simulation, of the number of contacts $\left(n_{1}\right)$ involving $\mathrm{OP}(\mathrm{RNA})$ atoms and $\mathrm{Na}$ (thin line, left $y$-axis) or Mg (thick line, right $y$-axis) ions ( $\left.d_{0}=0.28 \mathrm{~nm}\right)$. The vertical lines separate simulations with decreasing (from left to right) concentration of $\mathrm{Mg}$. exception of trajectories $\mathbf{2}$ and $\mathbf{6}$. Therefore, 2 trajectories over 6 display, in the first part of simulation $(\mathrm{Mg} \sim 10 \%$ of $\mathrm{Na})$, final values of $\mathrm{CN}_{n}$ higher than that of the crystallized 1OOA aptamerprotein complex. All trajectories display $\mathrm{CN}_{n}$ higher than the minimal value 0.002 (PDB 1B23). Concerning the second part (lower $\mathrm{Mg}$ concentration) of simulation, all of the trajectories end with $C N_{n}$ higher than the 1OOA threshold.

The second feature is more specific for each trajectory. Trajectory 1 reaches a higher value at high $\mathrm{Mg}$ concentration (first $10 \mathrm{~ns}$ ), then, after a sudden decrease due to the removal of phosphate-adsorbed $\mathrm{Mg}$ cations, the complex recovers high values of $C N_{n}$ when other $\mathrm{Mg}$ cations are adsorbed by RNA (see the corresponding plot in Figure 3). The complete removal of $\mathrm{Mg}$ cations (last $10 \mathrm{~ns}$ ) does not allow the unwrapping of OPN away from RNA. In trajectories $\mathbf{2}$ and $\mathbf{6}$, the slow sticking effect of phosphate adsorbed $\mathrm{Mg}$ ions is also displayed, with a final partial unwrapping of OPN away from RNA when all Mg ions are removed in trajectory 6 (last $10 \mathrm{~ns}$ ). These two trajectories display a OPN wrapping that becomes less tight, compared to the other trajectories, when no $\mathrm{Mg}$ is present. In complexes $\mathbf{3}$, 4 , and 5, higher $C N_{n}$ values are reached in the first stage of high $\mathrm{Mg}$ concentration and Mg removal has no apparent effect on the OPN wrapping. In trajectory 3 the partial recovery of phosphateadsorbed Mg occurs in the second simulation stage (central 10ns window) and the complete removal is again not sufficient to trigger the OPN unwrapping away from RNA, even though a partial unwrapping is displayed (last $10 \mathrm{~ns}$ ).

The behavior described above is also displayed by the non-electrostatic and non-solvation energy contribution to the formation of the OPN-RNA complex, i.e., the Lennard-Jones complexation energy. This is the sum of Lennard-Jones pair contributions to energy, with the sum extended to $i-j$ pairs, $i$ running over OPN atoms and $j$ running over RNA atoms. The time evolution of this contribution is displayed in the right panels of Figure 2. The initial values, close to zero, rapidly decrease to values lower than $-50 \mathrm{kcal} / \mathrm{mol}$ in all cases except trajectories 2 and 6, that are those not reaching the threshold for $C_{n}$. According to this observation, the value of $-50 \mathrm{kcal} / \mathrm{mol}$ for the Lennard-Jones complexation energy appears as a threshold for an irreversible wrapping. The correlation between the behavior of $\mathrm{CN}_{n}$ in the absence of $\mathrm{Mg}$ cations and that of the LennardJones complexation energy is displayed by trajectories $\mathbf{3}, \mathbf{4}$, and $\mathbf{5}$, i.e., those trajectories where the OPN wrapping is more evident and $\mathrm{Mg}$ cations are adsorbed by both OPN-R3 and OPN. In trajectories $\mathbf{3}$ and $\mathbf{4}$ there is partial unwrapping when no $\mathrm{Mg}$ is present, while in trajectory $\mathbf{5}$ the wrapping is recovered and the energy becomes lower than $-100 \mathrm{kcal} / \mathrm{mol}$.

The O-Mg contacts account for all the OPN and OPNR3 contacts with $\mathrm{Mg}$ in the second half of trajectories. Differences between $\mathrm{X}$ (with $\mathrm{X}$ any atom in the solute) and $\mathrm{O}=\mathrm{OP}, \mathrm{OD}, \mathrm{OE}$ (see Methods and Supplementary Material) as $\mathrm{Mg}$ adsorber, are displayed only in the first half of the trajectories, while in the second half there is no difference between the calculation including the addressed $\mathrm{O}(\mathrm{OP}, \mathrm{OD}$, and $\mathrm{OE}$ ) atoms and all the atoms in the OPN protein and RNA aptamer, respectively. This behavior shows that the OPN protein arranges its conformation to adapt to the constraint 
of possible $\mathrm{Mg}$-OP and $\mathrm{Mg}-\mathrm{OD} / \mathrm{OE}$ salt bridges. A detailed analysis of the salt bridges and charge neutralization for the 6 OPN-RNA complexes simulated for $10 \mathrm{~ns}$ is reported in Table 3.

The different rows for each trajectory display the number of contacts $n_{1}$ (see Supplementary Material for its definition), by decreasing $\mathrm{Mg}$ concentration (from top to bottom). The charge of OPN and RNA reported in the last two columns is computed by assigning one half of the ionic charge to OPN and RNA, respectively, when the ion is involved in a salt bridge of type OPN-M-RNA (the fifth column). It must be noticed that the negative charge of both partners in the complex, OPN and OPN-R3 aptamer, is minimal in the presence of $10 \%$ of Mg. A significant decrease of charge (the charge becomes more negative) occurs when $\mathrm{Mg}$ cations are removed from the system. The exchange of divalent cations between binding sites and the bulk is very slow in RNA, as it has not been observed in 200ns long MD simulations (Casalino et al., 2017). However, it can be observed that the initial approach between the two partners occurring when $\mathrm{Mg}$ is present, allows the replacement of missing $\mathrm{Mg}$ cations with Na cations, both in OPN and RNA. This process is similar to what has been observed for RNA (see Figure S7 and discussion in the Supplementary Material).

Summarizing, in all of the simulated trajectories 10-ns long and in the presence of about $10 \%$ of $\mathrm{Mg}$ cations among the positive counterions, the cations trigger the collapse of OPN onto the RNA aptamer, as shown by the initial time evolution of $C N_{n}$ (Figure 2). Then, unwrapping becomes difficult: unwrapping occurs only in one case (6) over six simulations during the following 2 simulations with decreasing number of $\mathrm{Mg}$ cations. In this case we notice that no efficient replacement of $\mathrm{Mg}$ by $\mathrm{Na}$ occurs in the adsorbed layer.

\subsubsection{Extending Simulations to $50 \mathrm{~ns}$}

Two selected simulations, $\mathbf{1}$ and $\mathbf{3}$, have been extended to $55 \mathrm{~ns}$ in the presence of $\mathrm{Na}$ and $\mathrm{Mg}$ counterions in the conditions of the simulated body fluid. In both simulations, the wrapping of OPN around OPN-R3 increases. The extent of wrapping is measured via the R quantity in Equation 2 in the Supplementary Material. In Figure 4, bottom panels, the time evolution of $\mathrm{R}$ shows that the wrapping is extended in both cases, but for trajectory $\mathbf{3}$ it is more extended than for $\mathbf{1}$.

This observation clearly shows that when the initial (the first 10 ns) wrapping is moderate (see Figure 2, trajectory 1), the wrapping is moderate also over a longer time-scale $(\mathrm{R} \sim 0.9)$. On the other hand, when the initial wrapping is large (trajectory 3 in Figure 2), the two partners continue the slow build-up of the wrapped assembly.

In Figure 4, top panels, the time evolution of the number of adsorbed cations ( $n_{1}, \mathrm{Na}$ in thin line, $\mathrm{Mg}$ in thick line) is displayed. Even if in both cases the number of $\mathrm{Mg}$ cations that is adsorbed is almost the total number of Mg ions in the sample (18 over 20 in both cases), the time evolution is markedly different in the two cases. In the first case (1) the Mg adsorption is not yet completed at $55 \mathrm{~ns}$ and consequently the number of adsorbed $\mathrm{Na}$ ions is still kept at the initial level $\left(n_{1}=15-16\right)$. In the second trajectory (3) the number of adsorbed $\mathrm{Na}$ ions is significantly decreased when the number of adsorbed $\mathrm{Mg}$ ions is stable at 18. This exchange between $\mathrm{Mg}$ and $\mathrm{Na}$ in the adsorbed layer $\left(d_{0}<0.28 \mathrm{~nm}\right)$ occurs when the wrapping of OPN around OPN-R3 displays values of $\mathrm{R}$ lower than 0.9 .

The time evolution of solvent accessible surface area (SASA) for OPN and OPN-R3 is also different in the two cases (data not shown). The SASA of RNA within the first 20 ns of simulation is very similar in the two cases, but during the last $20 \mathrm{~ns}$ the SASA(OPN-R3) for $\mathbf{3}$ is significantly larger than for $\mathbf{1}$, thus indicating a partial RNA unfolding upon the larger wrapping of 3. The time evolution of RMSD (Figure S8) displays, for both 55-ns trajectories, a similar behavior. The RNA fragment is kept within the same extent of deviation obtained after the first 10 ns. In trajectory $\mathbf{1}$, the fragment is even slightly restructured at the end of the trajectory, compared to trajectory 3 . The OPN $\mathrm{N}$-terminus becomes rigid after about $20 \mathrm{~ns}$ in both trajectories, and the C-terminus appears as following the collapse of the $\mathrm{N}$ terminus, with a time delay that is larger for $\mathbf{3}$ than for $\mathbf{1}$. In both trajectories, the C-terminus becomes more rigid after $40 \mathrm{~ns}$.

Following the analysis performed for the OPN-(OPN-R3) complex for the first $10 \mathrm{~ns}$ of simulation (Table 3), it is possible to measure the effective charge of the OPN-RNA complex accounting for the adsorption of $\mathrm{Mg}$ and $\mathrm{Na}$ cations within the first peak of the radial distribution function, that is within the distance $d_{0}=0.28 \mathrm{~nm}$. For trajectory $1,18 \mathrm{Mg}$ ions are adsorbed among the total amount of 20 , while only $16 \mathrm{Na}$ ions among the total amount of 366 are adsorbed. In case of trajectory 3, the number of adsorbed $\mathrm{Mg}$ ions is the same (see above), but that of $\mathrm{Na}$ ions is significantly smaller (11) when averaged over the last ns. Nevertheless, the final values of $n_{1}$ are similar, 16 and 15 for $\mathrm{Na}$ in, respectively, $\mathbf{1}$ and $\mathbf{3}$.

The calculation of $Q_{\text {eff }}$ (see Supplementary Material) gives -17.5 and -13.5 for OPN and OPN-R3, respectively, in case 1 , and -16.5 and -27.5 for 3 , respectively. In both cases the negative charge of the assembly is strongly screened at short distances: 37 and 53\%, respectively, of the total bare charge of $-44-39=-83$.

The visualization of the configurations of the complex OPN(OPN-R3) obtained at the end of trajectories $\mathbf{1}$ and $\mathbf{3}$ (Figure 5, left and right panels, respectively) shows several common features for the two trajectories.

Both final configurations display the formation of two nascent protein domains. One involves the $\mathrm{N}$-terminal region (first 110 residues), strongly interacting with the RNA aptamer via extended salt bridges involving both $\mathrm{Na}$ and $\mathrm{Mg}$ cations. Among the 20 available $\mathrm{Mg}$ cations, 15 (left panel) and 14 (right panel) ions are concentrated in the assembly of OPN-R3 with the $\mathrm{N}$-terminal OPN domain. The second domain (approximately the C-terminal 174 residues) contains a few cations (3 and 4, respectively, for $\mathbf{1}$ and $\mathbf{3}$ ). No $\mathrm{Cl}$ anion is adsorbed over the assembly. The loop in the stem-loop topology of RNA has different orientations, since in $\mathbf{1}$ the loop is directed toward the C-terminal domain of OPN (left panel), while the opposite is for 3 (right panel). This shows that the role of RNA sequence is in keeping the stem-loop RNA topology and the density of negative charges of phosphate groups, while the positions of bases have not a strong effect in the assembly topology. 
TABLE 3 | Number of ions ( $\mathrm{Na}$ and $\mathrm{Mg})$ within first-shell layer $\left(d_{0}<0.28 \mathrm{~nm}\right)$ from different sets of atoms: all atoms in OPN protein (OPN); all O atoms in carboxylate side-chains of $\mathrm{D}$ and $\mathrm{E}$ residues in OPN [O(D/E)]; all atoms in OPN-R3 aptamer (RNA); all non phosphodiester $\mathrm{O}$ atoms in phosphate groups of OPN-R3 (OP); complex indicates the OPN-(OPN-R3) complex.

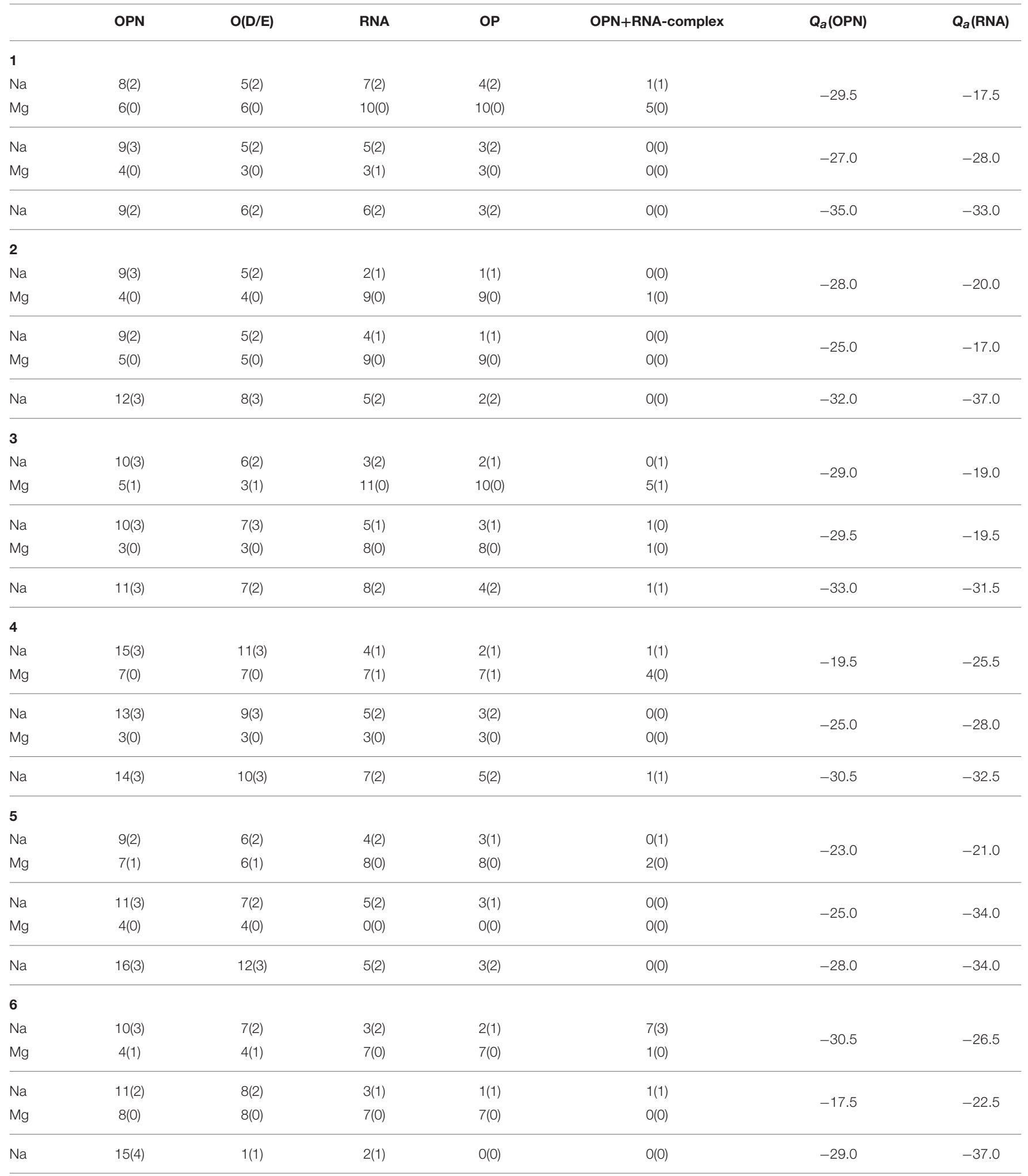

Root-mean square errors are within parentheses. Averages are over the last 5 ns of each 10-ns long trajectory. 

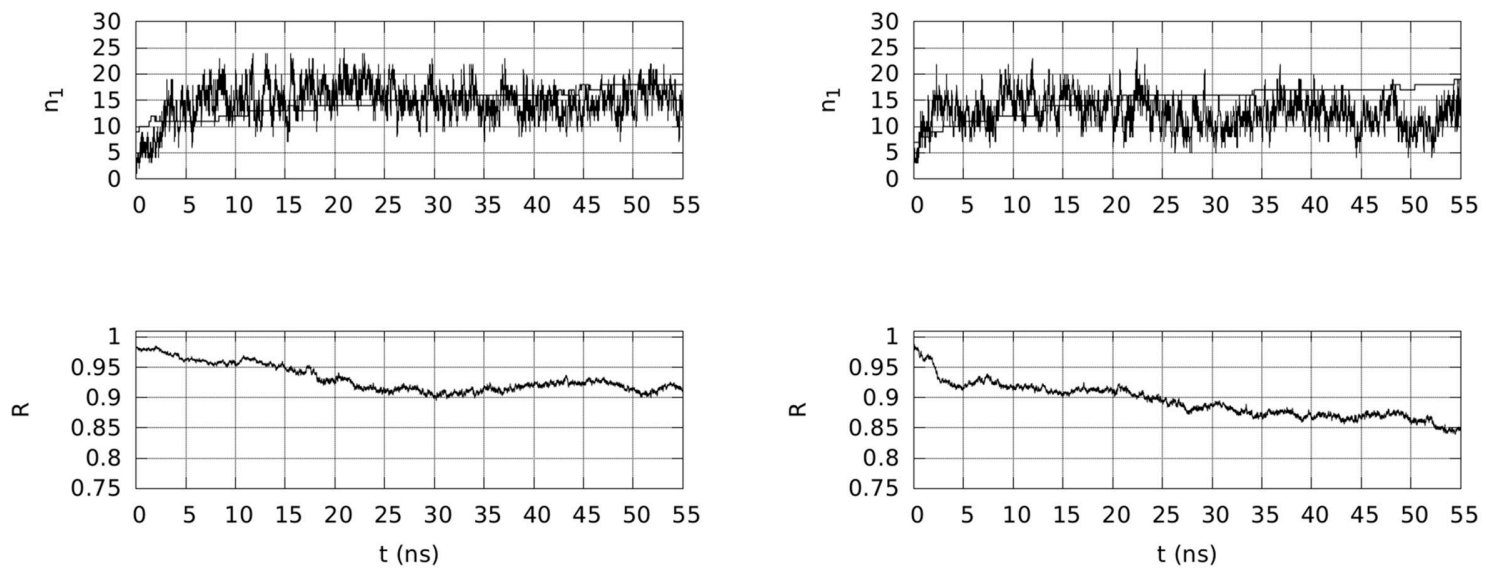

FIGURE 4 | Top: Time evolution, within 55 ns of simulation, of the number of contacts $\left(n_{1}\right)$ involving any atom in OPN and OPN-R3, and either Na (thin line) and Mg (thick line) ions $\left(d_{0}=0.28 \mathrm{~nm}\right)$. Bottom: Time evolution, in the same time-window, of R (Equation 2 in the Supplementary Material), describing the extent of assembly between OPN and its RNA aptamer. Left panels are for trajectory $\mathbf{1}$ and right panels for trajectory $\mathbf{3}$.
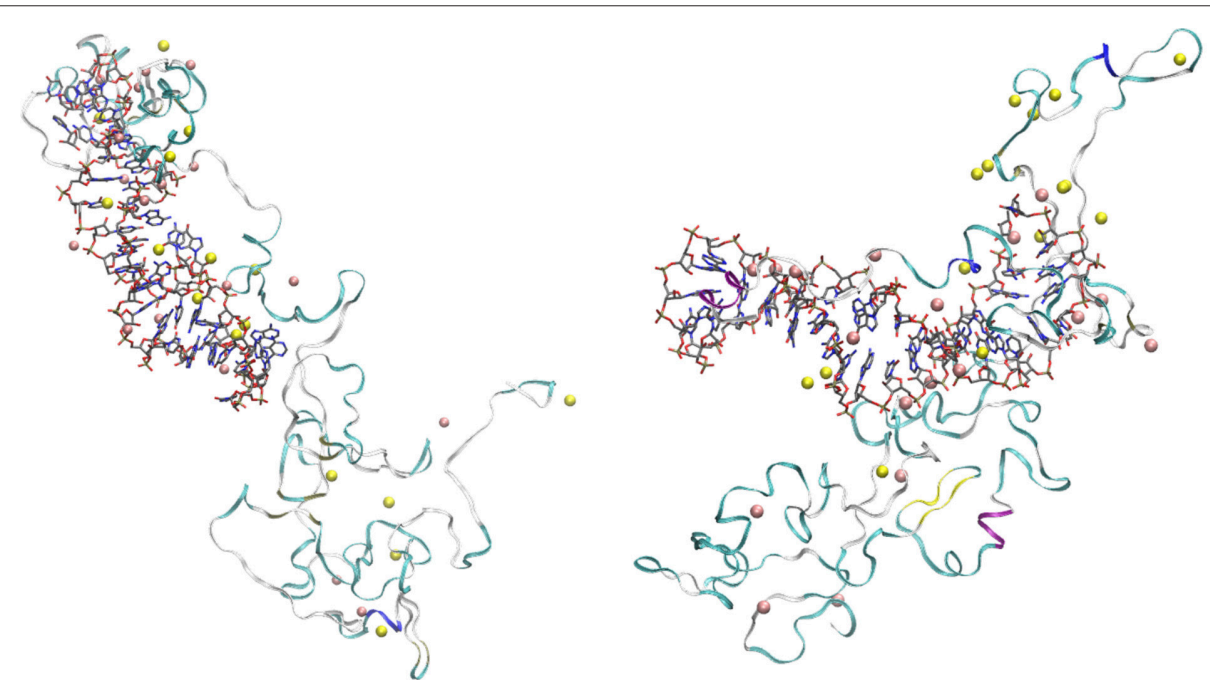

FIGURE 5 | Final configurations of OPN-(OPN-R3) complexes in trajectories $\mathbf{1}$ (left) and $\mathbf{3}$ (right). OPN-R3 (RNA) is represented as sticks. The RNA loop is in the center (left) and on the left-hand side (right). OPN protein is represented as a ribbon with colors associated to secondary motifs identified by the STRIDE (Frishman and Argos, 1995) algorithm ( $\alpha$-helix is in magenta and blue, $\beta$-sheet is yellow, turns are in cyano, coils in white); Na ions are yellow spheres, Mg are pink spheres. Atomic and bond radii are arbitrary. The ions were selected as those within a distance of $0.28 \mathrm{~nm}$ from any atom of the solute complex. The number of ions $\left(n_{1}\right)$ is 18 for $\mathrm{Mg}$ (both configurations) and, respectively, 15 and 16 for $\mathrm{Na}$ in trajectory $\mathbf{1}$ (left) and $\mathbf{3}$ (right).

Since the two trajectories are originated from different initial random OPN-(OPN-R3) contacts (Figure S1, Supplementary Material), the formation of the two domains in OPN appears as a common trend for the assembly: the N-terminus of OPN, rich in $\mathrm{D}$ and $\mathrm{E}$ residues, tends to strongly interact with cations adsorbed by the RNA aptamer; the C-terminus, more hydrophobic, is left for an autonomous protein folding, with a few cations involved. Despite the specificity of this type of assembly, similar longrange folding events are observed in simulations of DNA-protein complexes (Lambrughi et al., 2016). The structures displayed in Figure 5 are atomistic descriptions of the schematic drawings in Figure 1A.
This two-domain behavior of OPN, when the latter is exposed to its RNA aptamer OPN-R3, can be summarized as a folding-upon-binding mechanism, opposite to that proposed for the interaction between OPN and heparin in the absence of divalent cations. Nevertheless, the type of interaction between the OPN N-terminus and RNA is consistent with the few structural results reported in the literature for isolated OPN. The most recent NMR data for non-phosphorylated OPN (Platzer et al., 2015) are consistent with small deviations from random coil values, but the $\mathrm{N}$-terminus displays a long conformationally extended stretch. The propensity for such structural extension in the $\mathrm{N}$-terminus clearly assists 
the formation of the network of salt bridges displayed in Figure 5.

\section{CONCLUSIONS}

The interactions between OPN and its RNA aptamer OPNR3 are investigated by means of atomistic models where $\mathrm{Na}$ and $\mathrm{Mg}$ cations are included. The formation of diffuse layers of monovalent cations and more persistent layers of divalent cations around the phosphate groups of RNA, as it is reported in the literature, is confirmed. The negatively charged $\mathrm{N}$-terminus of OPN takes part to this interaction, with cations performing a strong screening of the large negative charge of the nascent assembly.

Several limitations affect the model: (i) the rough model of divalent cations; (ii) the short length of MD trajectories due to the slow conformational change of the disordered protein; (iii) the slow process of exchange of divalent cations. Despite these limitations, the two longer simulations, started from different initial configurations, describe the assembly as two nascent domains: (i) the ternary complex between OPN-R3, the Nterminus of OPN rich in negatively charged residues (D and E), and most of the divalent cations recruited in the solution; (ii) the C-terminus of OPN adopting a collapsed structure where cations are not involved and that points oppositely to the first domain.

This two-domain topology can be altered by OPN phosphorylation, the latter providing a negative charge to other residues (most commonly $\mathrm{S}, \mathrm{T}$, and $\mathrm{Y}$ ) that are more distributed among OPN regions (Lenton et al., 2017).

\section{REFERENCES}

Aaqvist, J. (1990). Ion-water interaction potentials derived from free energy perturbation simulations. J. Phys. Chem. 94, 8021-8024. doi: 10.1021/ j100384a009

Casalino, L., Palermo, G., Abdurakhmonova, N., Rothlisberger, U., and Magistrato, A. (2017). Development of site-specific $\mathrm{Mg}^{2+}$-rna force field parameters: A dream or reality? Guidelines from combined molecular dynamics and quantum mechanics simulations. J. Chem. Theory and Comput. 13, 340-352. doi: 10.1021/acs.jctc.6b00905

Christensen, B., Schack, L., Kläning, E., and Sørensen, E. S. (2010). Osteopontin is cleaved at multiple sites close to its integrin-binding motifs in milk and is a novel substrate for plasmin and cathepsin d. J. Biol. Chem. 285, 7929-7937. doi: 10.1074/jbc.M109.075010

Cisneros, G. A., Karttunen, M., Ren, P., and Sagui, C. (2014). Classical electrostatics for biomolecular simulations. Chem. Rev. 114, 779-814. doi: 10.1021/ cr300461d

Du, J., Hou, S., Zhong, C., Lai, Z., Yang, H., Dai, J., et al. (2008). Molecular basis of recognition of human osteopontin by $23 \mathrm{c} 3$, a potential therapeutic antibody for treatment of rheumatoid arthritis. J. Mol. Biol. 382, 835-842. doi: 10.1016/j.jmb.2008.07.075

Duarte, F., Bauer, P., Barrozo, A., Amrein, B. A., Purg, M., Åqvist, J., et al. (2014). Force field independent metal parameters using a nonbonded dummy model. J. Phys. Chem. B 118, 4351-4362. doi: 10.1021/jp501737x

Fennell, C. J., and Gezelter, J. D. (2006). Is the ewald summation still necessary? Pairwise alternatives to the accepted standard for long-range electrostatics. J. Chem. Phys. 124:234104. doi: 10.1063/1.2206581

Frangogiannis, N. G. (2012). Matricellular proteins in cardiac adaptation and disease. Physiol. Rev. 92, 635-688. doi: 10.1152/physrev.000 08.2011
However, this study confirms the importance of electrostatic interactions, showing the further importance of divalent cations in the change of OPN shape, in the location of the aptamer interface, and in the determination of a possible independent domain left open to other types of interactions. The newborn domains in the OPN-(OPN-R3) assembly may provide different functions to OPN, thus providing a general frame for the OPN plasticity.

\section{AUTHOR CONTRIBUTIONS}

All authors listed have made a substantial, direct and intellectual contribution to the work, and approved it for publication.

\section{ACKNOWLEDGMENTS}

This work has been performed within the Nanomax-Encoder MIUR flagship project of national research programme. We acknowledge the CINECA award under the ISCRA initiative, for the availability of high performance computing resources (projects NONIONS and BIONICS) and support. GLP thanks dr Angelo Perico (Cnr-ISMac) for the stimulating discussions.

\section{SUPPLEMENTARY MATERIAL}

The Supplementary Material for this article can be found online at: https://www.frontiersin.org/articles/10.3389/fchem. 2018.00002/full\#supplementary-material

Frishman, D., and Argos, P. (1995). Knowledge-based secondary structure assignment. Proteins 23, 566-579. doi: 10.1002/prot.340230412

Hu, D. D., Lin, E. C. K., Kovach, N. L., Hoyer, J. R., and Smith, J. W. (1995). A biochemical characterization of the binding of osteopontin to integrins $\alpha_{\nu} \beta_{1}$ and $\alpha_{\nu} \beta_{5}$. J. Biol. Chem. 270, 26232-26238. doi: 10.1074/jbc.270.44.26232

Humphrey, W., Dalke, A., and Schulten, K. (1996). Vmd visual molecular dynamics. J. Mol. Graphics 14, 33-38. doi: 10.1016/0263-7855(96)00018-5

Hunter, G. K., O’Young, J., Grohe, B., Karttunen, M., and Goldberg, H. A. (2010). The flexible polyelectrolyte hypothesis of protein-biomineral interaction. Langmuir 26, 18639-18646. doi: 10.1021/la100401r

Kahles, F., Findeisen, H. M., and Bruemmer, D. (2014). Osteopontin: a novel regulator at the cross roads of inflammation, obesity and diabetes. Mol. Metabolism 3, 384-393. doi: 10.1016/j.molmet.2014.03.004

Kalmar, L., Homola, D., Varga, G., and Tompa, P. (2012). Structural disorder in proteins brings order to crystal growth in biomineralization. Bone 51, 528-534. doi: 10.1016/j.bone.2012.05.009

Kurzbach, D., Canet, E., Flamm, A. G., Jhajharia, A., Weber, E. M. M., Konrat, R., et al. (2017). Investigation of intrinsically disordered proteins through exchange with hyperpolarized water. Angew. Chem. Int. Ed. 56, 389-392. doi: 10.1002/anie.201608903

Kurzbach, D., Platzer, G., Schwarz, T. C., Henen, M. A., Konrat, R., and Hinderberger, D. (2013). Cooperative unfolding of compact conformations of the intrinsically disordered protein osteopontin. Biochemistry 52, 5167-5175. doi: $10.1021 /$ bi400502c

Kurzbach, D., Schwarz, T. C., Platzer, G., Höfler, S., Hinderberger, D., and Konrat, R. (2014). Compensatory adaptations of structural dynamics in an intrinsically disordered protein complex. Angew. Chem. Int. Ed. 53, 3840-3843. doi: 10.1002/anie.201308389

Lambrughi, M., De Gioia, L., Gervasio, F. L., Lindorff-Larsen, K., Nussinov, R., Urani, C., et al. (2016). Dna-binding protects p53 from interactions with 
cofactors involved in transcription-independent functions. Nucleic Acids Res. 44, 9096-9109. doi: 10.1093/nar/gkw770

La Penna, G. (2003). A constrained maximum entropy method in polymer statistics. J. Chem. Phys. 119, 8162-8174. doi: 10.1063/1.1609197

La Penna, G., Morante, S., Perico, A., and Rossi, G. C. (2004). Designing generalized statistical ensembles for numerical simulations of biopolymers. J. Chem. Phys. 121, 10725-10741. doi: 10.1063/1.1795694

Lejeune, D., Delsaux, N., Charloteaux, B., Thomas, A., and Brasseur, R. (2005). Protein-nucleic acid recognition: statistical analysis of atomic interactions and influence of dna structure. Proteins 61, 258-271. doi: 10.1002/prot.20607

Lenton, S., Grimaldo, M., Roosen-Runge, F., Schreiber, F., Nylander, T., Clegg, R., et al. (2017). Effect of phosphorylation on a human-like osteopontin peptide. Biophys. J. 112, 1586-1596. doi: 10.1016/j.bpj.2017.03.005

Li, S., and Wang, L. (2012). Phosphorylated osteopontin peptides inhibit crystallization by resisting the aggregation of calcium phosphate nanoparticles. Cryst. Eng. Commun. 14, 8037-8043. doi: 10.1039/C2CE 26140E

Liu, J., and Lu, Y. (2006). Fast colorimetric sensing of adenosine and cocaine based on a general sensor design involving aptamers and nanoparticles. Angew. Chem. Intl. Ed. 118, 96-100. doi: 10.1002/ange.200502589

Mazzali, M., Kipari, T., Ophascharoensuk, V., Wesson, J., Johnson, R., and Hughes, J. (2002). Osteopontin-a molecule for all seasons. Q. J. Med. 95, 3-13. doi: $10.1093 /$ qjmed/95.1.3

Mi, Z., Guo, H., Russell, M. B., Liu, Y., Sullenger, B. A., and Kuo, P. C. (2009). Rna aptamer blockade of osteopontin inhibits growth and metastasis of mda-mb231 breast cancer cells. Mol. Therapy 17, 153-161. doi: 10.1038/mt. 2008.235

Momma, K., and Izumi, F. (2011). Vesta 3 for three-dimensional visualization of crystal, volumetric and morphology data. J. Appl. Crystallogr. 44, 1272-1276. doi: $10.1107 /$ S0021889811038970

Oyane, A., Kim, H.-M., Furuya, T., Kokubo, T., Miyazaki, T., and Nakamura, T. (2003). Preparation and assessment of revised simulated body fluids. J. Biomed. Mater. Res. 65A, 188-195. doi: 10.1002/jbm.a.10482
Peysselon, F., Xue, B., Uversky, V. N., and Ricard-Blum, S. (2011). Intrinsic disorder of the extracellular matrix. Mol. Biosyst. 7, 3353-3365. doi: 10.1039/C1MB05316G

Platzer, G., Żerko, S., Saxena, S., Koźmiński, W., and Konrat, R. (2015). 1H, 15N, 13C resonance assignment of human osteopontin. Biomol. NMR Assignments 9, 289-292. doi: 10.1007/s12104-014-9594-7

Plimpton, S. (1995). Fast parallel algorithms for short-range molecular dynamics. J. Comput. Phys. 117, 1-19. doi: 10.1006/jcph.1995.1039.

Ponta, H., Sherman, L., and Herrlich, P. A. (2003). Cd44: from adhesion molecules to signalling regulators. Nat. Rev. Mol. Cell. Biol. 4, 33-45. doi: $10.1038 / \mathrm{nrm} 1004$

Sodek, J., Ganss, B., and McKee, M. D. (2000). Osteopontin. Crit. Rev. Oral Biol. Med. 11, 279-303. doi: 10.1177/10454411000110030101

Want, J., Cieplak, P., and Kollman, P. A. (2000). How well does a restrained electrostatic potential (resp) model perform in calculating conformational energies of organic and biological molecules? J. Comput. Chem. 21, 1049-1074. doi: 10.1002/1096-987X(200009)21:12<1049::AID-JCC3>3.0. $\mathrm{CO} ; 2-\mathrm{F}$

Weber, G. F., Zawaideh, S., Hikita, S., Kumar, V. A., Cantor, H., and Ashkar, S. (2002). Phosphorylation-dependent interaction of osteopontin with its receptors regulates macrophage migration and activation. J. Leukocyte Biol. 72, 752-761. doi: 10.1189/jlb.72.4.752

Conflict of Interest Statement: The authors declare that the research was conducted in the absence of any commercial or financial relationships that could be construed as a potential conflict of interest.

Copyright (c) 2018 La Penna and Chelli. This is an open-access article distributed under the terms of the Creative Commons Attribution License (CC BY). The use, distribution or reproduction in other forums is permitted, provided the original author(s) and the copyright owner are credited and that the original publication in this journal is cited, in accordance with accepted academic practice. No use, distribution or reproduction is permitted which does not comply with these terms. 